\title{
Erratum to: Safety of long-term treatment with Pegvisomant: analysis of Spanish patients included in global ACROSTUDY
}

\author{
I. Bernabeu ${ }^{1}$ - A. Pico ${ }^{2}$ E. Venegas ${ }^{3}$ J. Aller $^{4}$ - C. Alvarez-Escolá ${ }^{5}$. \\ J. A. García-Arnés ${ }^{6} \cdot$ M. Marazuela ${ }^{7} \cdot$ P. Jonsson ${ }^{8} \cdot$ N. Mir ${ }^{9} \cdot$ M. García Vargas ${ }^{9}$. \\ Spanish ACROSTUDY Group ${ }^{10}$
}

Published online: 4 May 2016

(C) Springer Science+Business Media New York 2016

\section{Erratum to: Pituitary (2016) 19:127-137 DOI 10.1007/s11102-015-0691-0}

The authors of the article noticed an imported error in Table 6 of the published article.

In the Summary of PEG studies: Safety section, in the last row of Table 6 , while referring to the results, there are many values reported incorrectly, because they are actually corresponding to the absolute frequency.
The relative frequency the values should be, In AE treatment associated is $11.6 \%$ instead 23 (n) In SAE general, $15.56 \%$ instead of 31 In SAE treatment associated, $1.5 \%$ instead of 3 In Discont (all causes), $8 \%$ instead of 16 In Discont treat. associated, $1 \%$ instead of 2 In Liver dysfunction, $7 \%$ instead of 14 In Mortality, $5 \%$ instead of 10

The online version of the original article can be found under doi:10.1007/s11102-015-0691-0.

A. Pico

antonio.pico@goumh.umh.es

1 Complejo Hospitalario Universitario de Santiago,

Santiago de Compostela, Spain

2 Hospital General Universitario de Alicante, Alicante, Spain

3 Hospital Universitario Virgen del Rocío, Seville, Spain

4 Hospital Universitario Puerta de Hierro - Majadahonda, Madrid, Spain

5 Hospital Universitario La Paz, Madrid, Spain

6 Hospital Regional Universitario de Málaga, Málaga, Spain

7 Hospital Universitario de La Princesa, Madrid, Spain

8 Pfizer Endocrine Care, Sollentuna, Sweden

9 Pfizer Medical Department, Madrid, Spain

10 ACROSTUDY Centers, Madrid, Spain 